\title{
Kurzgutachten zur Zusammenarbeit zwischen öffentlichem Spital und der Pharmaindustrie vor dem Hintergrund des neuen Heilmittelgesetzes
}

\author{
J.-C. Rudin
}

\footnotetext{
1 s. auch Empfehlung Bundesamt für Sozialversicherung, BSV, vom 15. März 2002.

2 Art. 87 HMG.

3 Art. 33 Abs. 3 lit. a HMG.

4 Botschaft zur Revision des StGB, BBl 1999 S. 5536.

5 BBl 1999, S. 3453-7.
}

Korrespondenz:

lic. iur. Johann-Christoph Rudin Rechtsanwälte Spörri Peter Rudin Zollikerstrasse 4

CH-8032 Zürich

\section{Ausgangslage}

Im Zusammenhang mit dem Inkrafttreten des neuen HMG vom 1. Januar 2002 stellt sich für ein öffentliches Spital die Frage, inwieweit die bisherige Zusammenarbeit mit der Pharmaindustrie namentlich in den Bereichen Rabatte, Sponsoring und Zuwendung von Drittmitteln möglich und zulässig ist.

Spitäler haben bisher von Lieferanten von Pharmaka aufgrund der grossen Abnahmevolumina Rabatte erhalten. Diese Rabatte haben sich in der Kostenrechnung der öffentlichen Spitäler niederzuschlagen, sollten also in der Rechnung der Spitäler erfolgswirksam werden. Vorausgesetzt ist dabei, dass die Medikamente in der Spitalrechnung entsprechend den gewährten Rabatten zum reduzierten Preis berechnet werden. Zur Diskussion stehen sodann von medizinischen Komitees (z.B. von der American Heart Association oder Schweizerischen Gesellschaft für Innere Medizin) veranlasste Kongresse, an welchen Personen des Spitals eingeladen werden und an denen z. B. die Reise- und Unterkunftskosten für die Teilnehmer eines Spitals von der Pharmaunternehmen übernommen werden. Weiter führt das Spital für die Pharmaindustrie Studien durch, welche letztere finanziert. Schliesslich geht es auch um die Teilnahme an Kursen, welche durch die Pharmaindustrie finanziert werden (Spesen usw.) oder um Periodika, für welche die Pharmaindustrie aufkommt.

\section{Rechtsgrundlagen}

\section{Heilmittelgesetz}

Das seit Anfang 2002 geltende HMG schreibt in Art. 33 vor, dass Personen oder Organisationen, welche Arzneimittel verschreiben oder abgeben, dafür keine geldwerten Vorteile fordern oder annehmen dürfen. Es soll erreicht werden, dass die Abgabe von Heilmitteln ausschliesslich mit Blick auf den Patienten sowie medizinischer Indika- tionen und nicht beeinflusst von Zuwendungen der Pharmaindustrie erfolgt. Darüber hinaus stellt die Regelung klar, dass erhaltene Vergünstigungen an die Kostenträger weiterzugeben sind [1]. Verstösse gegen diese Vorschriften können mit Haft oder Busse bis zu Fr. 50 000.bestraft werden [2].

Durch die Gewährung geldwerter Vorteile von bescheidenem Wert sieht der Gesetzgeber dieses Ziel allerdings nicht gefährdet [3]. Darunter sind wohl analog der Regelung von Art. 322 ${ }^{\text {ter }}$ StGB (Bestechung) sozial übliche Geschenke zu verstehen, wie z. B. der Blumenstrauss für die Krankenschwester [4]. Das BSV bezieht sich in seiner Weisung vom 21. Dezember 2001 auf die Parlamentsdebatte sowie die bundesgerichtliche Rechtsprechung im Strafrecht, wonach Sachleistungen bis zu Fr. 300.- pro Firma, Empfänger (gemeint ist der Betrieb) und Jahr als bescheiden anzusehen sind. Zudem erklärt Art. 33 Abs. 3 lit. b HMG «handelsübliche und betriebswirtschaftlich gerechtfertigte Rabatte, die sich direkt auf den Preis auswirken» für zulässig.

Nach der Botschaft zum HMG [5] wird durch die Regelung von Art. 33 HMG insbesondere die Gewährung geldwerter Vorteile, wie z. B. Superboni, Reisen, Einladungen, Geschenke und Gratismuster, verboten, soweit sie im Hinblick auf die Verschreibung oder die Abgabe eines Arzneimittels erfolgen. Die eingangs erwähnten Kongresse, Kurse, Studien oder weiteren Zuwendungen werden durch diese Regelungen also dann tangiert, wenn sie die Beeinflussung im Hinblick auf die Verschreibung oder Abgabe eines Arzneimittels beabsichtigen oder bewirken.

\section{Arzneimittel-Werbeverordnung}

Dass sich der Gesetzgeber der wichtigen Bedeutung der Pharmaindustrie in der medizinischen Forschung und Entwicklung durchaus bewusst ist, zeigt Art. 11 der Arzneimittel-Werbeverordnung vom 17. Oktober 2001, in Kraft seit 1. Januar 2002. Danach hat sich der Repräsentationsaufwand im Zusammenhang mit wissen- 
schaftlichen Kongressen oder Promotionsveranstaltungen in einem vertretbaren Rahmen zu halten und muss in bezug auf den Hauptzweck der Veranstaltung von untergeordneter Bedeutung sein. Damit ist auch gesagt, dass die Durchführung von Kongressen usw. durch die Pharmaindustrie nicht als solches, sondern einzig die übermässige Repräsentation verpönt ist. In erster Linie sollen mit solchen Veranstaltungen wissenschaftliche Zwecke verfolgt werden. Dies liegt nicht zuletzt auch im Interesse des Staates, welcher dadurch erheblich weniger Ausgaben hat.

\section{Strafrechtliche Normen}

Mit der Zuwendung geldwerter Vorteile befasst sich seit dem 1. Mai 2000 auch das Strafgesetzbuch in Art. 322 $2^{\text {ter }} \mathrm{ff}$. Nach Art. 322 quater StGB wird die passive Bestechung, in Art. $322^{\text {sexies }}$ allgemein die Annahme von Vorteilen im Hinblick auf die Amtsführung unter Strafe gestellt. Dabei gilt es $\mathrm{zu}$ beachten, dass Drittmittel und Sponsoring mit der geltenden Fassung des StGB nicht grundsätzlich problematisiert werden sollen. Ein öffentliches Spital darf solche Zuwendungen auch unter dem neuen StGB entgegennehmen, sie müssen jedoch dem Spital oder einer Abteilung und nicht einer einzelnen Person zugute kommen [6, 7]. Dabei ist selbstverständlich nicht zu übersehen, dass auch ein formell korrekt gewährter Vorteil letztendlich auf Umwegen einer Einzelperson zugute kommen kann und neben den «offiziellen» Vorteilen auch private Anreize gewährt werden können. Solches Verhalten widerspricht klar dem Grundgedanken des Gesetzes, welches die Unabhängigkeit des Amtsträgers gewährleisten will. Um entsprechende Umgehungen zu verhindern, empfiehlt es sich, die vorgeschlagenen spital- und fakultätsinternen Regelungen betreffend die Geringfügigkeit von Geschenken weit zu fassen und sämtliche Vorteilsgewährungen einzuschliessen [7]. Notwendig erscheint darüber hinaus jedoch eine klare strukturelle Trennung der Kompetenzen. Durch die Spitalorganisation ist die Abgabe und Anwendung der Medikamente personell getrennt vom Einkauf zu regeln. Die mit dem Einkauf betraute Person soll unabhängig von Eigeninteressen den grösstmöglichen Vorteil für die Institution bzw. schliesslich den Patienten und den Krankenversicherer im Auge haben.

Straffrei ist die Annahme geringfügiger Geschenke durch Einzelpersonen, soweit diese sozial toleriert sind und aufgrund gesellschaftlicher Normen keine Befangenheit hervorrufen können [8]. Die diesbezüglich erwähnten internen Regelungen zur Handhabung von Geschenken sind zweifelsohne wünschenswert [7]. Diese Ge- setzesbestimmung stellt jedoch kein Novum dar, sahen doch die kantonalen Personalgesetze bereits entsprechende Regelungen vor. Mit der nunmehr auf Bundesebene bestehenden Regelung ist jedoch eine einheitliche Handhabung gewährleistet, was zweifelsohne zu begrüssen ist. Als Höchstwert gelten somit analog der bundesgerichtlichen Rechtsprechung im Strafrecht gesamtschweizerisch Fr. 300.- pro Betrieb und Jahr. Restriktivere kantonale Regelungen aufgrund des Personalgesetzes beanspruchen dagegen weiterhin Geltung.

\section{Personalrecht}

Alle Personalrechte statuieren ausdrücklich oder implizite ein Verbot zur Annahme von Geschenken oder anderer Vergünstigungen. Ausgenommen sind Höflichkeitsgeschenke von geringem Wert. Bei Zweifeln über die Geringfügigkeit entscheidet die vorgesetzte Dienststelle über die Zulässigkeit der Annahme.

Diese Regelungen führen gegenüber dem Heilmittelgesetz und den strafrechtlichen Regelungen jedoch kaum zu relevanten weiteren Einschränkungen. Das Verbot richtet sich wie bei den Bestechungsstraftatbeständen an die einzelnen natürlichen Personen.

\section{Zulässigkeit von Zuwendungen}

\section{Allgemeines}

$\mathrm{Zu}$ unterscheiden ist grundsätzlich zwischen Rabatten, Sponsoring und der Vergabe von Drittmitteln. Rabatte stellen einen Preisnachlass dar, welcher beispielsweise aufgrund einer dauerhaften Geschäftsbeziehung oder der Menge gewährt wird. Während beim Sponsoring geldwerte Vorteile ohne direkte gleichwertige Gegenleistung gewährt werden, werden durch die Vergabe von Drittmitteln direkte Gegenleistungen z. B. in der Form von Forschungsarbeiten von der Pharmaindustrie gekauft [7].

\section{Rabatte}

Die Höhe der gewährten Rabatte wirkt sich infolge Pauschalisierung von Diagnosen und Therapien bei Allgemein- und Halbprivatpatienten nicht direkt auf die Leistungen der Krankenversicherer aus, führen aber durch die Berücksichtigung in der Kostenrechnung zu einer günstigeren Tarifstruktur des Spitals. Bei Privatpatienten vergüten die Krankenversicherer in Vorwegnahme von gewährten Vergünstigungen sogar nur $90 \%$ der Medikamentenpreise, egal, ob Rabatte effektiv gewährt werden oder nicht. Die Aushandlung von Rabatten ist den Leistungs- 
erbringern daher nicht zu verwehren und liegt sofern korrekt weitergegeben - im Sinne des Gesetzes. Die Vergünstigungen schlagen sich auf diese Weise in der Tarifstruktur nieder. Dieses Vorgehen entspricht der Regelung in Art. 33 Abs. 3 lit. b HMG, welcher handelsübliche und betriebswirtschaftlich gerechtfertigte Rabatte, die sich direkt auf den Preis auswirken, für zulässig erklärt.

In der Botschaft des Bundesrates zu einem Bundesgesetz über Arzneimittel und Medizinprodukte (Heilmittelgesetz, HMG) vom 1. März 1999 wird zu Art. 33 namentlich ausgeführt, dass die Beeinflussung von Fachpersonen (Ärzte, Apotheker, Drogisten), welche Arzneimittel anwenden oder abgeben, durch geldwerte Vorteile, wie zum Beispiel (Zitat) «Superboni, Reisen, Einladungen, Geschenke, Gratismuster etc.», verboten werden soll. Dieses Verbot soll aber «handelsübliche und betriebswirtschaftlich gerechtfertigte Rabatte, die sich direkt auf den Preis auswirken», nicht verhindern. Preisvorteile, so die Botschaft, sollen im wettbewerblichen Umfeld möglich sein. In Konkretisierung der «direkten Auswirkung auf den Preis» führt die Botschaft sodann aus, dass «sie entweder dem selbstzahlenden Patienten direkt zugute kommen, oder aber sich indirekt [9] - via Rabatte an die Krankenversicherer - in der Prämienhöhe niederschlagen».

Adressat des Verbotes, geldwerte Vorteile entgegenzunehmen oder zu fordern, sind «Fachpersonen, welche Arzneimittel anwenden oder abgeben». In der Spitalorganisation sollten wie bereits erwähnt dort, wo dies noch nicht der Fall ist, die Abgabe und Anwendung der Medikamente durch das behandelnde Spitalpersonal vom Einkauf der Medikamente völlig getrennt werden. Es ist darauf zu achten, dass die arzneimittelabgebenden Ärzte und Fachpersonen weder direkt noch indirekt irgendwelche Vorteile aus der Abgabe von Medikamenten erzielen können. Diejenigen Personen, welche für ihr Spital Rabatte fordern, sind wiederum nicht direkt mit der Abgabe von Arzneimitteln an den einzelnen Patienten zu betrauen.

Hinzuweisen ist hier auf den Umstand, dass Patienten, welche nach dem Spitalaufenthalt auf die Einnahme von (teuren) Medikamenten angewiesen sind, möglicherweise nicht mehr auf ein anderes Medikament wechseln, obwohl nun kein Rabatt mehr gewährt wird und deshalb der volle Preis zu Buche schlägt. Diesem Risiko ist auch mit den vorgeschlagenen organisatorischen und strukturellen Massnahmen nicht zu begegnen. Dies hat zur Folge, dass unter Umständen ein gegenüber anderen Produkten teureres Medikament zum vollen Preis vom Patienten bzw. dem Krankenversicherer zu berappen ist. Allerdings sind diese Folgen durch die Möglichkeit der Abgabe von Generika, welche meist deutlich preisgünstiger sind, nicht als gravierend einzuschätzen. Wollte man dieses Kostenrisiko gänzlich verhindern, müsste man den Leistungserbringern vorschreiben, welche Medikamente sie zu verabreichen hätten. Ebendies wurde in der Volksabstimmung vom 4. März 2001 abgelehnt. Ein Verbot von Rabatten steht nach dem Gesagten ebenfalls nicht zur Debatte. Es bleibt daher die Feststellung, dass Preisnachlässe bei Grossabnehmern üblich und auch im Gesundheitswesen unter den gegebenen Voraussetzungen insbesondere der Weitergabe der Rabatte - unter dem Strich zu begrüssen sind. Rabatte aber beispielsweise in der Form von Rückvergütungen einer anderen Rechnung als der betreffenden Spitalrechnung gutzuschreiben, verletzt klarerweise das HMG.

\section{Sponsoring (Verpflegungs-, Unterkunfts- und Reisekosten sowie Rahmenprogramm an Kursen und Kongressen, Periodika usw.)}

In den Bereich des Sponsorings fallen nach der genannten Definition von medizinischen Komitees durchgeführte Kongresse und Kurse, an welche Personen von öffentlichen Spitälern eingeladen werden und bei denen die Pharmaindustrie die Kosten der Teilnehmer für Reise, Unterkunft und Verpflegung sowie für das Rahmenprogramm übernimmt. Eine direkte Gegenleistung erhält die Pharmaindustrie hierfür nämlich nicht. Gleiches gilt für Periodika, welche durch ein Pharmaunternehmen finanziert werden. Es liegt nahe, dass sich ein Pharmaunternehmen damit in die Gunst einer arzneimittelabgebenden Person stellen will und kann. Soweit an den fraglichen Veranstaltungen eine Mehrzahl von Teilnehmern aus verschiedenen Institutionen eingeladen ist und die Teilnahme keine Übernahme von Pflichten mit sich bringt, erscheint die Gefahr einer Beeinflussung durch die blosse Teilnahme zwar nicht ausgeschlossen, aber in einem vertretbaren Rahmen. Dies gilt jedenfalls dann, wenn sich das Pharmaunternehmen an die Regelung von Art. 11 der Arzneimittel-Werbeverordnung hält.

Die kostenlose Verpflegung anlässlich der Veranstaltung ist m.E. als geringfügige Zuwendung zu betrachten. Demgegenüber ist die Übernahme weiterer durch die Veranstaltung verursachter Spesen, wie z.B. Reisespesen, Übernachtung usw., problematischer, ist sie doch umfangmässig viel bedeutsamer. Der Gefahr einer Beeinflussung 
könnte hier dadurch begegnet werden, dass die verschiedenen Pharmaunternehmen unabhängig von einzelnen Veranstaltungen Beiträge an das Spital bzw. einen von diesem verwalteten Fonds leisten, welcher ausschliesslich zur Übernahme solcher Spesen zur Verfügung steht, dessen Mittel jedoch von der Spitalleitung nach eigenem Ermessen eingesetzt werden können. Auf diesem Weg könnten auch Periodika oder Internetzugänge gesponsert werden. Damit wäre neben der Einhaltung von Art. 33 HMG zudem die Gefahr einer Bestrafung wegen passiver Bestechung oder Vorteilsannahme im Sinne von Art. 322 quater $u$ und Art. $322^{\text {sexies }}$ StGB beseitigt, da Empfänger der Zuwendung keine Einzelperson, sondern eine Institution ist. Die Sammlung sämtlicher Zuwendungen in einem Fonds brächte den Vorteil, dass die Zuordnung der Leistungen an einzelne Pharmaunternehmen nicht mehr gemacht werden könnte und daher auch keine Beeinträchtigung bei der Verschreibung oder Abgabe zu befürchten ist. Dabei würden einzelne Pharmaunternehmen die bisher als Sponsoring erbrachten Leistungen als freiwillige Beiträge in einen Fonds einbringen. Dieser Fonds würde verwendet, um die Reise-, Unterkunfts- und Verpflegungskosten von Fachtagungsteilnehmern zu finanzieren. Die Verwendung der Fondsmittel wäre durch ein Reglement näher zu umschreiben, so dass die Pharmaunternehmen absehen können, wie ihre Beiträge verwendet werden. Wesentlich wäre ebenfalls die klare Organisation der Fondsleitung, welche unabhängig von der Pharmaindustrie Ausschüttungen im Rahmen des Reglementes machen könnte.

Eine gewisse Entschärfung des Problems wäre auch dadurch zu erreichen, dass beispielsweise das Flugticket dem Fachtagungsteilnehmer nicht mehr direkt vom Pharmaunternehmen, sondern indirekt von der Spitalverwaltung zugesandt wird, welche ihr Visum darauf anzubringen hätte. So wäre zumindest gewährleistet, dass die Spitalverwaltung die Übersicht über die erbrachten Leistungen behält.

Was das Werbematerial angeht, das an Personen des Spitals abgegeben wird, kann auf den Richtwert von Fr. 300.- pro Jahr verwiesen werden. Darüber hinausgehende Leistungen liessen sich mit Art. 33 HMG nicht mehr rechtfertigen.

Hinsichtlich des Rahmenprogramms von Veranstaltungen ist im Auge zu behalten, dass dieses gegenüber dem wissenschaftlichen Hauptzweck der Veranstaltung jedenfalls nur eine untergeordnete Rolle spielen darf, was beispielsweise bei der kostenlosen Verpflegung anzunehmen ist. Dagegen sprengen m.E. separate Veranstaltungen, wie z. B. Reisen oder Unterhaltungs- programme am Rande der Veranstaltung, welche nicht unmittelbar durch diese veranlasst werden, den Rahmen des Vertretbaren. Die Unabhängigkeit des Teilnehmers in bezug auf seine Tätigkeit in einem öffentlichen Spital erscheint in solchen Fällen gefährdet. Durch die Annahme solcher Zuwendungen bewegt sich eine Person im Bereich der strafbaren Handlungen. Es ist zu bedenken, dass im Einzelfall kaum nachzuweisen sein wird, ob sich eine Person tatsächlich beeinflussen hat lassen, weshalb einzig auf die erhaltene Zuwendung und deren Eignung zur Beeinflussung abzustellen ist. Der Umweg über Beiträge an einen Fonds ist für diesen Zweck nicht praktikabel. Der Veranstalter, welcher ein attraktives Rahmenprogramm gestaltet, möchte um die Gunst des Teilnehmers werben, was unter dem neu geltenden Heilmittelgesetz gerade verpönt ist bzw. nur in sehr beschränktem Umfang erlaubt wird [10].

\section{Drittmittel}

Die Gefahr der Beeinflussung der Arzneimittelabgabe durch die Pharmaindustrie besteht zwar in viel grösserem Masse beim Sponsoring, ist aber auch bei Drittmitteln, für welche die Pharmaindustrie einen Gegenwert erhält, nicht auszuschliessen. Es handelt sich dabei beispielsweise um Zuwendungen der Pharmaindustrie für Studien oder Forschungsaufträge. Soweit für solche Zuwendungen eine entsprechende Gegenleistung durch das Spital erbracht wird, welche in einem realistischen Verhältnis zur erhaltenen Zuwendung steht, ist dagegen nichts einzuwenden. Dies umso mehr, als jede Studie durch eine unabhängige Ethikkommission genehmigt wird, welche u.a. festlegt, wer was finanziert. Damit ist bereits eine gewisse Aufsicht gewährleistet. Vorausgesetzt ist selbstredend, dass die Finanzierung nicht an weitere Nebenpflichten geknüpft wird, welche die Verschreibung oder Abgabe von Arzneimitteln beeinträchtigen. Um sich dem Vorwurf einer Beeinflussung zu entziehen und insbesondere, um den Straftatbeständen der Bestechung zu entgehen, wäre es auch hier ratsam, wenn die entsprechenden Beträge nicht einzelnen Personen, sondern dem Spital bezahlt würden [11]. Im Lichte der neuen Gesetzgebung nicht zulässig wären indes Beiträge der Pharmaindustrie, welche zur erhaltenen Gegenleistung in einem klaren Missverhältnis stehen und auf diesem Wege eine ungerechtfertigte Zuwendung bedeuteten.

Diesen Ausführungen bleibt anzumerken, dass ein äusserst wirksames Mittel gegen den Vorwurf der unzulässigen Beeinflussung durch die Pharmaindustrie sowie gegen Bestechungs-
11 vgl. dazu Botschaft zur Revision des StGB, BBl 1999, S. 5537 
vorwürfe die Publizität bzw. Transparenz von erhaltenen Leistungen ist. Wer sich aus lauteren Motiven an der Forschung und Entwicklung sowie der kostengünstigen Preisentwicklung im Gesundheitswesen beteiligt, kann gegen eine Offenlegung nichts einzuwenden haben.

\section{Fazit}

Das Forderung und Erhalten von Preisnachlässen beim Einkauf der Spitalapotheken ist vor dem Hintergrund von Art. 33 HMG nicht verboten, solange die Rabatte nicht einzelnen Fachpersonen zugute kommen, sondern sich erfolgswirksam auf die Rechnung des Spitals auswirken. Unter dieser Prämisse machen sich die Verantwortlichen für den Einkauf von Arzneimitteln nicht strafbar, wenn sie Rabatte fordern und in der Folge dem Spital im gleichen Ausmass Rabatte gewährt werden.

Die Zusammenarbeit der Spitäler mit der Pharmaindustrie im Bereich Forschung und Entwicklung, Sponsoring usw. ist weiterhin möglich und sogar erwünscht, soweit dadurch nicht die Verschreibung oder Abgabe von Arzneimitteln gefördert werden soll. Dafür ist einzig die medizinische Indikation massgebend. Werden geldwerte Vorteile ausgehandelt, so sind diese in jedem Fall an die Kostenträger weiterzugeben. Ausgenommen sind bescheidene Zuwendungen bis zu Fr. 300.- pro Betrieb und Jahr. Naturgemäss ist die Weitergabe von Vorteilen, welche durch die Kostenübernahme bei Kongressen, Kursen, Periodika o.ä. erfolgen, nicht möglich. Nachdem solche Veranstaltungen jedoch der notwendigen Weiterbildung des medizinischen Personals dienen und letztlich zu Kosteneinsparungen bei der öffentlichen Hand führen, liegen sie ebenfalls im öffentlichen Interesse. Übersteigen die geldwerten Zuwendungen indes den Aufwand für die erwünschte Aus- und Weiterbildung oder Forschung, so ist diese mit der neuen Gesetzgebung nicht zu vereinbaren. Um der Gefahr einer passiven Bestechung oder Vorteilsannahme im Sinne des Strafgesetzbuches zu entgehen, empfiehlt es sich sodann, möglichst keine Leistungen direkt an Einzelpersonen fliessen zu lassen, sondern wenn immer möglich an das Spital. Schliesslich dient der Verwirklichung des Grundgedankens des neuen Gesetzes auch eine möglichst weitreichende Offenlegung der Finanzierungen. 